\title{
纳米材料的生长用于含 $\mathrm{Cr}(\mathrm{VI})$ 纳米废渣的处理
}

\author{
刘炜珍 ${ }^{(1}$, 许新江 $^{(1)}$ ，王永净 ${ }^{(1)}$ ，何中 ${ }^{(2)}$, 卓楠 ${ }^{(2)}$, 黄丰 ${ }^{(1)}$, 林璋 $^{(1 *}$ \\ (1) 中国科学院福建物质结构研究所, 中国科学院光电材料化学与物理重点实验室, 福州 350002 ; \\ (2) 福建省(屏南)榕屏化工有限公司, 福建屏南 352300 \\ *联系人, E-mail: zlin@,fjirsm.ac.cn
}

2009-04-13 收稿, 2009-08-28 接受

中国科学院知识创新工程重要方向项目(编号: KJCX1.YW.07)、国家重点基础研究发展计划(编号: 2010CB933501)、国家自然科学青年基金 (批准号: 20803082)、国家杰出青年科学基金(批准号: 50625205)和福建省环保局科技计划项目(编号: 闽环科函 2007 第 43 号)资助

摘要 由于特殊的结构与性能, 纳米材料在各个领域被广泛使用. 纳米材料的环境行为、生物和生 态效应受到了极大关注. 另外, 一些工业生产实践中也产生纳米污染物. 然而, 关于如何控制和治 理纳米污染物的研究较少. 在前期的研究中, 通过控制纳米颗粒快速长大以消除纳米污染物环境 危害的思路被提出, 并应用于处理氯酸盐行业的含 $\mathrm{Cr}(\mathrm{VI})$ 纳米废渣. 在此基础上, 本文进一步分析 和对比了含 $\mathrm{Cr}(\mathrm{VI})$ 纳米废渣的高温和室温两种处理途径的相关物相变化特征和无害化机制, 选择 高温处理工艺路线进行了优化, 并进一步对无害化处理后固体粉末的物相、形貌、粒度、热性能 等性质进行了研究, 将其试用于陶瓷釉料. 该工作是纳米技术用于环保污染治理与资源化利用的 一个范例, 具有理论意义和实践价值.

\section{关键词 纳米材料 环境 污染物 重金属 纳米颗粒 快速生长 回收利用}

纳米材料，由于其高比表面积、高吸附效率、高 催化活性等优异的性能, 广泛地应用于各个领域 $[1,2]$, 包括吸附废水中的重金属 ${ }^{[3.4]}$ 和降解有机物的环保领 域 $[5,6]$. 然而近期研究发现本身无毒的物质在尺寸达 到纳米级以后, 可能因其高的吸附性和表面活性而 产生新的生理毒性 ${ }^{[7,8]}$; 有毒的重金属物质在尺寸达 到纳米级以后, 其迁移性和反应性增加, 导致生物毒 性增强 [9]. 因此, 纳米材料的广泛应用可能会带来新 类型的环境问题. 另外, 一些工业生产实践中也常常 产生纳米废弃物, 这些对环境有危害的纳米物质统 称为纳米污染物 10$]$.

纳米污染物的毒理和生物效应 [11], 以及纳米物 质在环境中的转化规律 ${ }^{[12]}$ 是目前国际上的研究热点, 然而如何控制和消除纳米污染物危害性的研究较少. 为了从源头上减少纳米污染物的危害性, Huang等人 研究了各种回收装置, 例如静电集尘和静电过滤技 术 ${ }^{[13]}$ 、催化氧化和连续再生装置 ${ }^{[14]}$. Morawska等人 ${ }^{[15]}$
发现, 用过滤器将空气中的微小粒子移除的效率只 有 $40 \%$, 如果是纳米颗粒移除率更低, 而且收集到的 纳米废弃物也难以处理. Liu等人 ${ }^{[16]}$ 提出消除纳米污 染物的最好办法之一，是通过人工控制纳米颗粒快 速长大以消除其高吸附性、高反应活性及生理毒性. 理论上纳米材料都是热力学亚稳相, 可自发长大, 然 而其生长过程在动力学上可能十分缓慢, 使得纳米 污染物可以在自然界长期存在. 因此, 通过人工控制 纳米材料快速长大以实现纳米污染物的无害化是很 有必要的.

例如，氯酸盐行业产生一种吸附了重金属 $\mathrm{Cr}(\mathrm{VI})$ 的纳米废渣. 由于纳米材料高吸附性, 吸附的六价铬 难以用传统的方法洗脱, 但是通过自然淋浸六价铬 又会慢慢渗入地表, 污染地下水、江河、湖泊, 危害 农田、水产和人类健康. 目前主要处理方法是还原固

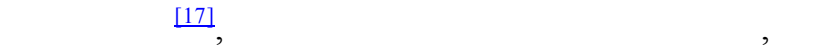
可能造成二次污染. 由于环境中的三价铬和六价铬 
可以相互转化, 而且长期存在于自然环境中的含铬 纳米颗粒一旦暴露在空气中, 很可能通过迁移成为 大气中的有毒纳米污染物或者水体中的污染物. 因 此, 如何有效处理含 $\mathrm{Cr}(\mathrm{VI})$ 纳米废渣而不造成二次污 染是极其有意义的. 在前期的工作中, 我们利用在纳 米材料生长热力学和动力学上的研究基础 ${ }^{[18 ~ 20]}$, 针 对氯酸盐行业产生的含 $\mathrm{Cr}(\mathrm{VI})$ 纳米废渣的处理难题, 找到了一种矿化剂 $\left(\mathrm{NaHCO}_{3}\right)$ 促使含 $\mathrm{Cr}(\mathrm{VI})$ 纳米废渣 中的纳米材料进行物相转变和快速生长, 从而实现 $\mathrm{Cr}(\mathrm{VI})$ 的有效分离、回收以及纳米废弃物的无害化处 理 ${ }^{[16]}$.

本文进一步分析和对比了含 $\mathrm{Cr}(\mathrm{VI})$ 纳米废渣的 高温和室温两种处理途径及其相关机制. 通过开展 高温条件下含 $\mathrm{Cr}(\mathrm{VI})$ 纳米废渣处理工艺路线的优化 研究, 以及脱毒固体粉末的性质和综合利用研究, 证 实了这一技术的工业可行性.

\section{1 实验}

\section{1 试剂与仪器}

$\mathrm{NaHCO}_{3} 、 \mathrm{Na}_{5} \mathrm{P}_{3} \mathrm{O}_{10}$ 、造纸级高岭土、羧甲基纤 维素钠 $(\mathrm{CMC})$ 等试剂均为工业级. 仪器: TCS-PC-150 台秤、B20 型搅拌器、30L 不锈钢桶、YXQ-LS-50SII 恒温压力锅、SSC450 型离心机、烘箱、QHPE400×600 颚式破碎机、QHZM-1 振动球磨机.

\section{2 高温处理工程优化}

原渣含水率约为 $50 \%$, 主要成分为 $\mathrm{Mg}(\mathrm{OH})_{2}$, $\mathrm{CaCO}_{3}$ 以及 $\mathrm{NaCl}, \mathrm{KClO}_{3}$ 等可溶性盐, $\mathrm{Cr}(\mathrm{VI})$ 含量约为 $2407 \mathrm{mg} / \mathrm{kg}$. 称取原渣 5 10 kg 放入搅拌机, 按 $1: 2 \sim 1: 5$ 的固液比加入 $5 \sim 25 \mathrm{~L}$ 水, 充分搅拌形成均匀 的悬浮浆料, 再加入 $2 \sim 5 \mathrm{~kg} \mathrm{NaHCO}_{3}$ 混合均匀. 将混 合好的浆料倒入不锈钢桶中, 连同不锈钢桶放入恒 温压力锅, 加热到 $120^{\circ} \mathrm{C}$ 并保温 $1 \sim 5 \mathrm{~h}$. 高压后将不锈 钢桶取出冷却, 可发现原先糊状的浆料分成了两层, 即澄清的 $\mathrm{Cr}(\mathrm{VI})$ 上层清液和沉降在底部的固体层. 分 离出上层清液, 取一些上层清液测 $\mathrm{Cr}(\mathrm{VI})$ 浓度. 将处 理后的固体渣用水离心洗涤 5 遍后, 干燥、粉碎、球 磨、过篮、装袋, 即为脱毒固体粉末. 取一些脱毒固 体粉末样品, 进行 XRD 物相分析、粒度分析、DTA 和浸出 $\mathrm{Cr}(\mathrm{VI})$ 浓度测试.

\section{3 脱毒固体粉末部分替代水晶釉实验}

该实验在瓷砖生产线上进行，图 1 给出相应的操
作流程. 将各原料按照以下成分配比: 水晶釉熔块 $65 \% \sim 85 \%$, 脱毒固体粉末 $5 \% \sim 20 \%$, 造纸级高岭土 $5 \%$ 、三聚磷酸钠 $0.3 \%$ 、羧甲基纤维素钠 $0.1 \%$. 取 $300 \mathrm{~g}$ 混合料, 加 $200 \mathrm{~mL}$ 水, 球磨 $30 \mathrm{~min}$. 将球磨后的浆料 过篮, 慢慢搅拌让气泡排出, 静置一段时间. 之后将 浆料均匀地淋在经过 $1000^{\circ} \mathrm{C}$ 素烧的坏面上, 再将坏 料放到 $1130^{\circ} \mathrm{C}$ 烧结工段烧制成产品.

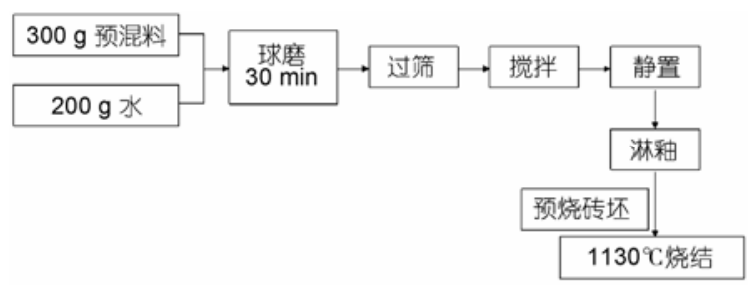

图 1 脱毒固体粉末部分替代水晶釉实验的操作流程

\section{4 测试分析}

$X$ 射线衍射(XRD)测试是在 PANalytical X'Pert 型粉末衍射仪上进行, 铜靶 $\mathrm{Cu} \mathrm{K} \alpha, \lambda=0.15418 \mathrm{~nm}$, 管电压 $40 \mathrm{kV}$, 管电流 $40 \mathrm{~mA}$, 扫描范围 $2 \theta$ 为 $5^{\circ} \sim 85^{\circ}$, 步长 $0.017^{\circ}$, 数据采集时间每步 $10 \mathrm{~s}$. 固体样品的形 貌及尺寸采用 JSM-6700F 型扫描电子显微镜(SEM) 观察. 脱毒固体粉末的粒度分布采用 BT-9300S 型激 光粒度分析仪测试. 样品的 DTA 曲线采用 DTA404PC 高温差示扫描量热仪进行测量. 固体样品 的总铬含量检测采用等离子发射光谱仪(ICP-AES, Jobin Yvon Ultima2), 水溶液中的 $\mathrm{Cr}(\mathrm{VI})$ 浓度采用二 苯碳酰二肼分光光度法 $(\mathrm{GB} / \mathrm{T}$ 15555.4) 在日本岛津 UV-2550 分光光度计上测定. 固体 $\mathrm{Cr}(\mathrm{VI})$ 浸出采用国 家标准固体废物浸出毒性浸出方法一硫酸硝酸法或 醋酸缓冲溶液法(HJ/T299-2007, HJ/T300-2007).

\section{2 结果与讨论}

\section{1 处理方法及机制}

在前期氯酸盐行业含 $\mathrm{Cr}(\mathrm{VI})$ 纳米废渣的处理过 程中, 我们已经对高温条件下纳米废渣的物相转变、 快速生长和 $\mathrm{Cr}(\mathrm{VI})$ 分离的机制进行了研究 ${ }^{[16]}$, 通过 控制纳米材料快速生长使 $\mathrm{Cr}(\mathrm{VI})$ 脱附和回收，从而实 现含 $\mathrm{Cr}(\mathrm{VI})$ 纳米废渣的环保治理. 图 2(a)给出了高温 处理条件下纳米氢氧化镁物相转变、生长与 $\mathrm{Cr}(\mathrm{VI})$ 脱 附、分离的关系示意图. 在高温条件下，废渣中的氢 氧化镁纳米片首先转变成水菱镁矿纳米片的聚集体 导致 $\mathrm{Cr}(\mathrm{VI})$ 的脱吸附, 随后水菱镁矿聚集体转变成 

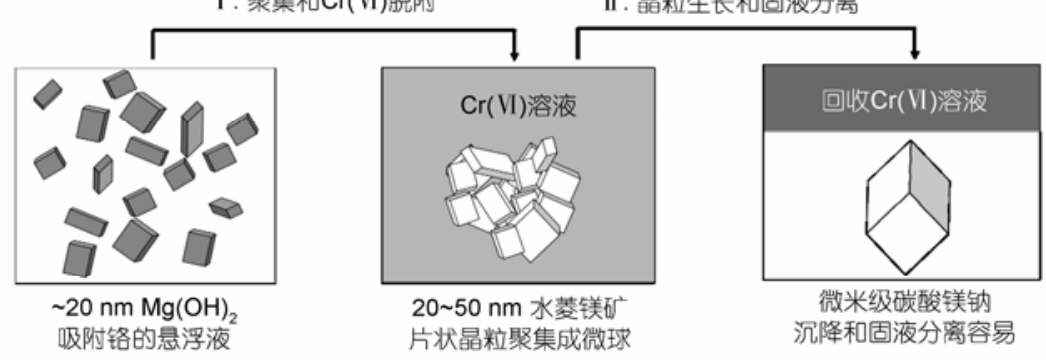

(b) 室温处理

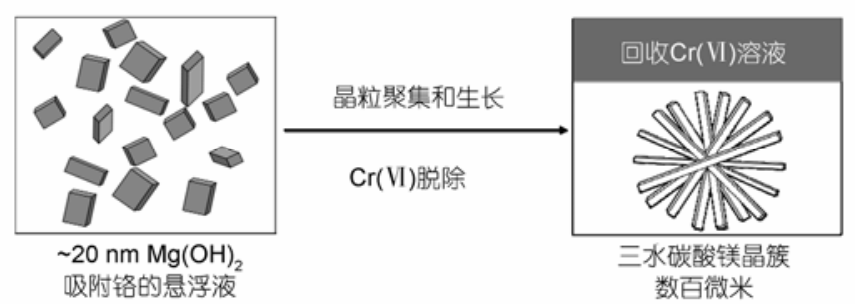

图 2 高温和室温处理中物相转变、生长和 $\operatorname{Cr}(\mathrm{VI})$ 分离机制

粒径为几微米的碳酸镁钠晶粒并进一步生长完善, 有利于固液分离. 为了降低处理成本、节约能耗, 我 们发展了室温处理方法. 研究发现, 室温条件下纳米 氢氧化镁的物相转变和生长出现了完全不同于高温 处理的现象. 如图 2(b)所示, 在室温处理条件下纳米 氢氧化镁转变成了数百微米的三水碳酸镁晶簇, 以 实现 $\mathrm{Cr}(\mathrm{VI})$ 的脱附和固液快速分离. 其中室温条件下 纳米氢氧化镁的物相变化、生长与 $\mathrm{Cr}(\mathrm{VI})$ 脱附、分离 的机制, 我们正在做进一步研究.

\section{2 工艺路线优化}

目前室温处理后的固体 $\mathrm{Cr}(\mathrm{VI})$ 浸出大于 2.63 $\mathrm{mg} / \mathrm{L}$, 还不能达到综合利用标准 $(\mathrm{HJ} / \mathrm{T}$ 301-2007), 而 高温处理后的固体 $\mathrm{Cr}(\mathrm{VI})$ 浸出小于 $0.5 \mathrm{mg} / \mathrm{L}$, 可以 满足要求. 因此, 我们选择高温处理途径进行工艺优 化. 图 3 为优化后的工艺路线, 优化实验采用半自动 化机械操作, 每批可处理 7 10 kg 含 $\mathrm{Cr}(\mathrm{VI})$ 废渣. 实 验得到最佳的处理条件为: 固液比 $1: 4, \mathrm{NaHCO}_{3}$ 用量 为每千克原渣 $0.4 \mathrm{~kg}$, 加热到 $120^{\circ} \mathrm{C}$ 保温 $4 \mathrm{~h}$. 处理后, $\mathrm{Cr}(\mathrm{VI})$ 上层清液可回收利用; 固体渣经过洗涤、烘干、 粉磨加工成脱毒固体粉末，进一步进行综合利用. 洗 涤水循环使用可以节约用水, 离心机第 1 遍洗涤水混 合下一批含 $\mathrm{Cr}(\mathrm{VI})$ 废渣进行高温处理, 可回收更多 $\mathrm{Cr}(\mathrm{VI})$ 并减少含 $\mathrm{Cr}(\mathrm{VI})$ 废水的处理成本.

\section{3 脱毒粉末的综合利用研究}

图 4 和表 1 将处理前后的样品进行了对比. 可见,

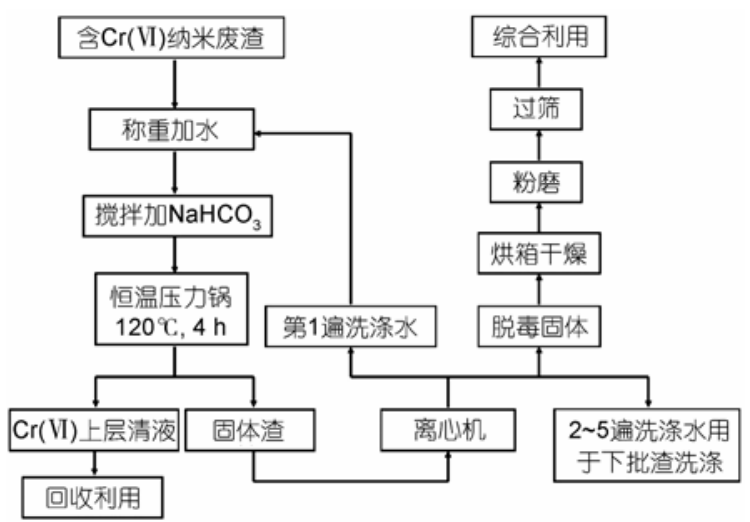

图 3 优化的高温处理工艺流程

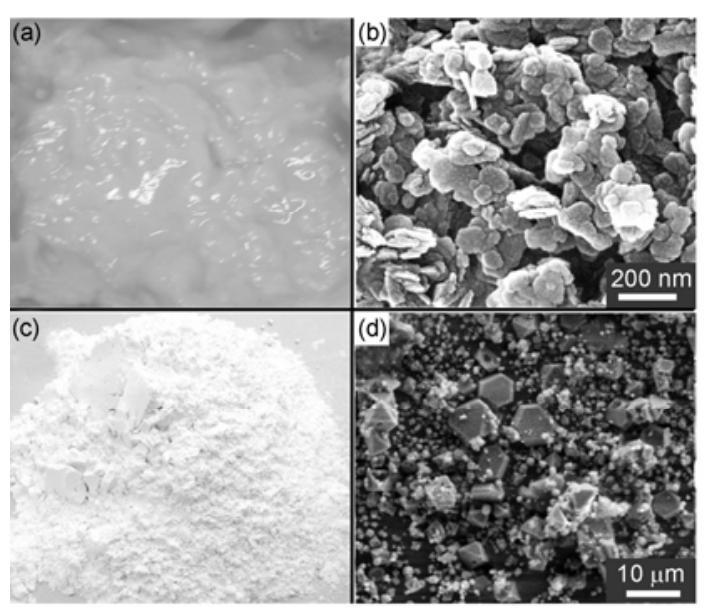

图 4 处理前后实物图及 SEM 图

(a) 原渣样品, (b) 原渣的 SEM, (c) 处理后样品, (d) 处理后 样品的 SEM 
表 1 处理前后样品对比

\begin{tabular}{ccccccc}
\hline 样品 & 主要物相组成 & $\mathrm{pH}$ 值 & 平均粒径 & 总铬含量 & $\begin{array}{c}\text { 上层清液 } \mathrm{Cr}(\mathrm{VI}) \text { 浓 } \\
\text { 度 } / \mathrm{mg} \cdot \mathrm{L}^{-1}\end{array}$ & $\begin{array}{c}\text { 骎出 } \mathrm{Cr}(\mathrm{VI}) \text { 浓度 } \\
/ \mathrm{mg} \cdot \mathrm{L}^{-1}\end{array}$ \\
\hline 原渣 & $\begin{array}{c}\mathrm{CaCO}_{3}, \mathrm{Mg}(\mathrm{OH})_{2} \\
\mathrm{CaCO}_{3}, \mathrm{Na}_{2} \mathrm{Mg}\left(\mathrm{CO}_{3}\right)_{2}\end{array}$ & 13 & $100 \mathrm{~nm}$ & 2407 & 121.5 & 132.6 \\
脱毒固体 & $\mathrm{Mg}_{5}\left(\mathrm{CO}_{3}\right)_{4}(\mathrm{OH})_{2} \cdot 4 \mathrm{H}_{2} \mathrm{O}$ & 9 & $9.0 \mu \mathrm{m}$ & 51.6 & 608.2 & 0.218 \\
\hline
\end{tabular}

高温处理方法将黄色黏稠的含 $\mathrm{Cr}(\mathrm{VI})$ 废渣变成了白色的 粉末(图 4(a)和(c)), $\mathrm{Cr}(\mathrm{VI})$ 分离效率达到了 97.8\%. 处理 前后样品的粒径发生了明显的变化(图 4(b)和(d)), 由于 纳米级的 $\mathrm{Mg}(\mathrm{OH})_{2}$ (图 4(b)) 变成了微米级的 $\mathrm{Mg}_{5}\left(\mathrm{CO}_{3}\right)_{4}(\mathrm{OH})_{2} \cdot 4 \mathrm{H}_{2} \mathrm{O}$ 聚集体和 $\mathrm{Na}_{2} \mathrm{Mg}\left(\mathrm{CO}_{3}\right)_{2}$ 晶粒(图 4(d)). 由表 1 可见, 处理后的固体粉末 $\mathrm{pH}$ 值比原渣低, 物相主要由 $\mathrm{CaCO}_{3}, \mathrm{Na}_{2} \mathrm{Mg}\left(\mathrm{CO}_{3}\right)_{2}$ 和 $\mathrm{Mg}_{5}\left(\mathrm{CO}_{3}\right)_{4}(\mathrm{OH})_{2}$. $4 \mathrm{H}_{2} \mathrm{O}$ 组成, 浸出 $\mathrm{Cr}(\mathrm{VI})$ 浓度为 $0.218 \mathrm{mg} \cdot \mathrm{L}^{-1}$, 已达到 综合利用标准(HJ/T 301-2007). 图 5 给出了脱毒固体 粉末的粒度分布, 测得样品的中位径为 $8.25 \mu \mathrm{m}$, 平 均粒径为 $9.0 \mu \mathrm{m}$, 图中可见脱毒固体粉末尺寸分布 窄, 粒度均一。

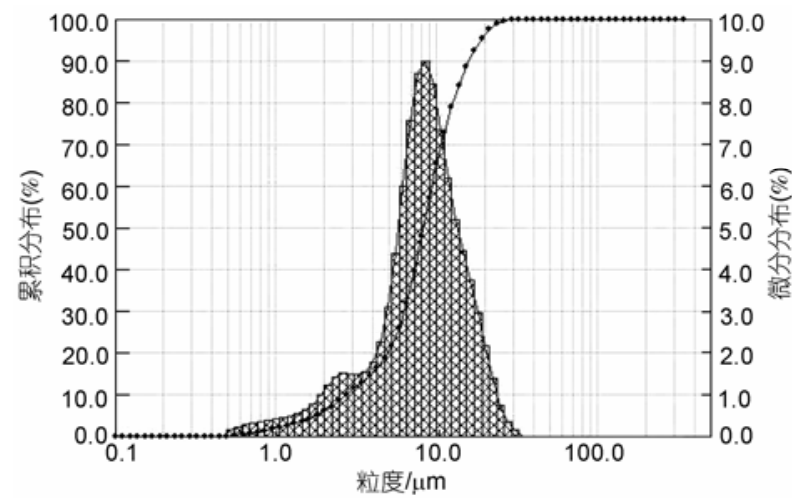

图 5 脱毒固体粉末粒度分布图

图 6 给出了脱毒固体粉末以 $10^{\circ} \mathrm{C} / \mathrm{min}$ 的升温速 率从 $100^{\circ} \mathrm{C}$ 到 $1000^{\circ} \mathrm{C}$ 的 DTA 曲线图. 可见, 脱毒固体 粉末主要有 3 个分解吸热峰. 第一个吸热峰温度范围 在 $400 \sim 600^{\circ} \mathrm{C}$, 峰值为 $525^{\circ} \mathrm{C}$, 这是 $\mathrm{Na}_{2} \mathrm{Mg}\left(\mathrm{CO}_{3}\right)_{2}$ 和 $\mathrm{Mg}_{5}\left(\mathrm{CO}_{3}\right)_{4}(\mathrm{OH})_{2} \cdot 4 \mathrm{H}_{2} \mathrm{O}$ 的分解吸热峰; 第二个吸热峰 温度范围在 $600 \sim 700^{\circ} \mathrm{C}$, 峰值为 $620^{\circ} \mathrm{C}$, 这是 $\mathrm{MgCO}_{3}$ 的分解吸热峰; 第三个吸热峰温度范围在 $800 \sim 920^{\circ} \mathrm{C}$, 峰值为 $880^{\circ} \mathrm{C}$, 这是 $\mathrm{CaCO}_{3}$ 分解吸热峰. 由 DTA 分析 可见, 镁碳酸盐的分解温度和碳酸钙的分解温度相 差 $100^{\circ} \mathrm{C}$ 左右, 可以利用二者分解温度的差别, 使脱 毒固体粉末中的镁碳酸盐充分分解而又避免其中碳
酸钙的分解，从而得到活性氧化镁和惰性碳酸钙的 混合粉末，用于制备镁水泥等. 由于脱毒固体粉末的 主要组成是镁钙碳酸盐, 而且粒度细小，可以利用其 受热分解产生微小气泡的特点生产多孔陶瓷、泡沫玻 璃等, 可广泛应用于冶金、化工、环保、能源、生物 等领域, 如用作金属熔体过滤、高温烟气净化、催化 剂载体和化工精滤材料等.

图 7 为脱毒固体粉末以不同比例替代水晶釉熔 块的瓷砖样品照片, 以考察脱毒固体粉末的性质和 在陶瓷釉料中的作用. 从图中可以看出, 脱毒固体粉 末代替 $5 \%$ 水晶釉熔块(图 7(b)), 釉面光泽没有明显

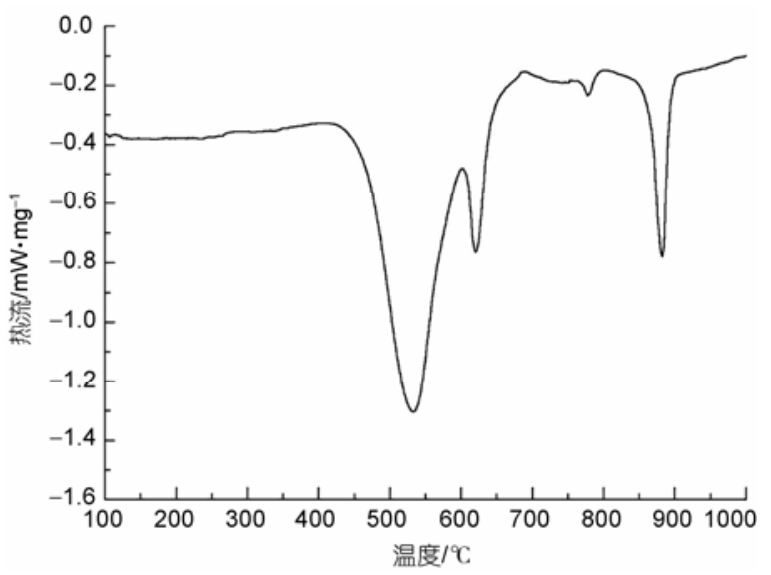

图 6 脱毒固体粉末 DTA 分析

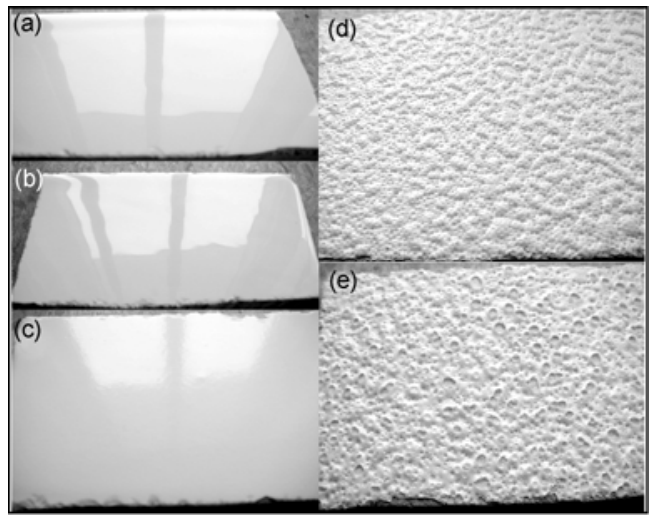

图 7 脱毒固体粉末以不同比例替代水晶釉熔块的瓷砖样品 (a) $0 \%$, (b) $5 \%$, (c) $10 \%$, ,d) $15 \%$, (e) $20 \%$ 
改变; 代替 10\%(图 7(c)), 釉面开始失去光泽出现亚 光效果, 说明脱毒固体粉末提高了熔体的秥度; 代替 15\%(图 7(d)), 釉面出现密麻的小鼓泡, 说明脱毒固 毒固体粉末使熔体黏度增加导致气泡排出困难. 代 替粉末分解产气时釉面已经开始熔融, 而且由于代 替 20\%(图 7(e)), 釉面鼓泡明显增大、破裂, 裂口锋利, 说明烧成结束时釉面还没有完全熔融. 可见, 脱毒固 体粉末有助熔作用, 可降低釉料的始熔温度, 增大釉 料熔融的温度范围, 提高熔体黏度. 因此, 脱毒固体 粉末可以添加在陶瓷亚光釉或水晶釉的配方中, 而 且利用其颗粒细小出现均匀小泡的特点还可以产生 个性化的陶瓷釉面.

\section{3 结论}

(1) 本文总结了采用控制纳米材料快速生长使
含 $\mathrm{Cr}(\mathrm{VI})$ 纳米废渣的 $\mathrm{Cr}(\mathrm{VI})$ 脱附和回收的高温和常温 处理途径, 以及它们的物相转变、生长和 $\mathrm{Cr}(\mathrm{VI})$ 分离 机制.

(2) 进行了含 $\mathrm{Cr}(\mathrm{VI})$ 纳米废渣的高温处理工艺优 化, 确定了最佳处理条件和工艺路线.

(3) 对含 $\mathrm{Cr}(\mathrm{VI})$ 纳米废渣处理后得到的脱毒固体 粉末进行了综合利用开发, 通过考察脱毒固体粉末 的物相、形貌、粒度、热性能等性质及其在水晶釉配 方中的作用，说明脱毒固体粉末可以应用于开发高 附加值的材料.

(4) 含 $\mathrm{Cr}(\mathrm{VI})$ 纳米废渣的处理工艺路线优化和脱 毒固体粉末的综合利用研究, 证明了通过控制纳米 材料生长进行纳米污染物治理的工业可行性. 本工 作为消除纳米污染物的环境危害并实现废渣的综合 利用提供了一个很好的范例.

\section{参考文献}

1 Gupta A K, Gupta M. Synthesis and surface engineering of iron oxide nanoparticles for biomedical applications. Biomaterials, 2005, 26: 3995-4021[DOI]

2 Chen X, Schluesener H J. Nanosilver: A nanoproduct in medical application. Toxicol Lett, 2008, 176: 1-12[DOI]

3 Ponder S M, Darab J G, Mallouk T E. Remediation of $\mathrm{Cr}(\mathrm{VI})$ and $\mathrm{Pb}$ (II) aqueous solutions using supported, nanoscale zero-valent iron. Environ Sci Technol, 2000, 34: 2564-2569[DOI]

4 Huang S H, Chen D H. Rapid removal of heavy metal cations and anions from aqueous solutions by an amino-functionalized magnetic nano-adsorbent. J Hazard Mater, 2009, 163: 174-179 $\underline{\text { DOI] }}$

5 Mauter M S, Elimelech M. Environmental applications of carbon-based nanomaterials. Environ Sci Technol, 2008, 42: 5843$5859 \underline{[\mathrm{DOI}]}$

6 Pouretedal H R, Norozi A, Keshavarz M H, et al. Nanoparticles of zinc sulfide doped with manganese, nickel and copper as nanophotocatalyst in the degradation of organic dyes. J Hazard Mater, 2009, 162: 674-681[DOI]

7 Hardman R. A toxicologic review of quantum dots: Toxicity depends on physicochemical and environmental factors. Environ Health Persp, 2006, 114: 165-172

8 Singh S, Nalwa H S. Nanotechnology and health safety-toxicity and risk assessments of nanostructured materials on human health. J Nanosci Nanotechno, 2007, 7: 3048-3070[1DOI]

9 Suzuki Y, Kelly S D, Kemner K M, et al. Radionuclide contamination-nanometre-size products of uranium bioreduction. Nature, 2002, 419: $134 \underline{[\mathrm{DOI}]}$

10 Tang H X. Environmental nano-pollutants (ENP) and their micro-interfacial processes on the aquatic particles. Acta Scientiae Circumstantiae, 2003, 32: 146-155

11 Hassellov M, Readman J W, Ranville J F, et al. Nanoparticle analysis and characterization methodologies in environmental risk assessment of engineered nanoparticles. Ecotoxicology, 2008, 17: 344-361 [DOI]

12 Christian P, von der Kammer F, Baalousha M, et al. Nanoparticles: Structure, properties, preparation and behaviour in environmental media. Ecotoxicology, 2008, 17: 326-343 $\underline{\text { [DOI] }}$

13 Huang S H, Chen C C. Ultrafine aerosol penetration through electrostatic precipitators. Environ Sci Technol, 2002, 36: 46254632 [DOI] 
14 Holmen B A, Ayala A. Ultrafine PM emissions from natural gas, oxidation- catalyst diesel, and particle-trap diesel heavy-duty transit buses. Environ Sci Technol, 2002, 36: 5041-5050[DOI]

15 Morawska L, Thomas S, Gilbert D, et al. A study of the horizontal and vertical profile of submicrometer particles in relation to a busy road. Atmos Environ, 1999, 33: 1261-1274 [DOI]

16 Liu W Z, Huang F, Liao Y Q, et al. Treatment of Cr- VI -containing $\mathrm{Mg}(\mathrm{OH})_{2}$ nanowaste. Angew Chem Int Edit, 2008, 47: 5619$5622[\mathrm{DOI}]$

17 Moon D H, Wazne M, Dermatas D, et al. Long-term treatment issues with chromite ore processing residue (COPR): Cr ${ }^{6+}$ reduction and heave. J Hazard Mater, 2007, 143: 629-635[DOI]

18 Huang F, Zhang H Z, Banfield J F. Two-stage crystal-growth kinetics observed during hydrothermal coarsening of nanocrystalline ZnS. Nano Lett, 2003, 3: 373-378[DOI]

19 Zhang J, Lin Z, Lan Y Z, et al. A multistep oriented attachment kinetics: Coarsening of ZnS nanoparticle in concentrated $\mathrm{NaOH}$. J Am Chem Soc, 2006, 128: 12981-12987 $\underline{\text { DOI] }}$

20 Zhang J, Wang Y H, Zheng J S, et al. Oriented attachment kinetics for ligand capped nanocrystals: Coarsening of thiol-PbS nanoparticles. J Phys Chem B, 2007, 111: 1449-1454ㅁD] 